\title{
DC-CIK biotherapy for a recurrent benign lymphoepithelial lesion of the salivary gland: A case report and literature review
}

\author{
DEJUN XING* and YUEMING ZHAO* \\ Department of Medical Oncology, Jilin Cancer Hospital, Changchun, Jilin 130012, P.R. China
}

Received April 2, 2014; Accepted August 5, 2014

DOI: $10.3892 /$ etm.2014.1937

\begin{abstract}
A benign lymphoepithelial lesion (BLEL) is an idiopathic inflammation of the parotid gland, characterized by hyperplasia, lymphocyte infiltration and the formation of epimyoepithelial islands, as well as atrophy of the gland parenchyma. Common treatment methods include immunosuppression and glucocorticoid therapies, in addition to surgical dissections. Cytokine-induced killer (CIK) cells sensitized to specific antigens by dendritic cells (DCs) are used in DC-CIK biotherapy. The present study reports the case of a 22-year-old female suffering from a postoperative recurrent BLEL on the left parotid gland, which was gradually increasing. Following initial unsuccessful conservative treatment attempts, a 10-day course of DC-CIK therapy was initiated, after which the lesion in the gland area was reduced in size and local infection and skin ulcerations were improved. DC-CIK biotherapy was continued for three months (four sessions of 10-day treatments with a 10-day break in between) until the lesion disappeared and the skin ulceration was healed. Computerized tomography scans of the parotid gland revealed complete remission of the primary lesion and recovery of the bone destruction. The patient was discharged and remained stable with no sign of recurrence during a 10 -month follow-up period. In the present case report, a successful DC-CIK adoptive cellular immunotherapy treatment for a BLEL was described for the first time.
\end{abstract}

\section{Introduction}

Benign lymphoepithelial lesions (BLELs) show three histological characteristics: Hyperplasia and infiltration of lymphocytes into the gland, atrophy of the gland parenchyma

Correspondence to: Professor Dejun Xing or Miss. Yueming Zhao, Department of Medical Oncology, Jilin Cancer Hospital, 1018 Huguang Road, Changchun, Jilin 130012, P.R. China

E-mail: xingdejun011614@163.com

E-mail: 81911654@qq.com

${ }^{*}$ Contributed equally

Key words: benign lymphoepithelial lesion, dendritic cell, cytokine-induced killer cell, adoptive cellular immunotherapy and hyperplasia and infiltration of epimyoepithelial islands in the gland duct. In 1952, Godwin denominated the condition as 'benign lymphoepithelial lesion' (1), which was newly classified as a benign salivary gland tumor in 1991 (2). BLELs were not considered to be tumors, but rather a type of reactive hyperplasia, characterized by a benign, long progression, which was not life-threatening and was rarely malignant in patients. BLELs appear mainly in unilateral or bilateral parotid tissues and the submandibular gland; however, cases of lymphoepithelial lesions of the salivary gland are rare, comprising $<3 \%$ of benign salivary gland tumors. At present, no standard therapy regimen is available for BLELs; however, glucocorticoid therapy, such as the administration of cyclophosphamide with prednisone, as an alternative to surgery has been reported $(3,4)$. A recent study demonstrated that BLELs are sensitive to radiotherapy, which may be beneficial in recurrent lesions (5). Although surgery and radiation are the main treatment methods (6-9), BLELs of the salivary gland have been rarely studied and no common therapy criteria or adoptive cellular immunotherapy have been reported.

\section{Case report}

A 22-year-old female was admitted to the Jilin Cancer Hospital (Changchun, China) in May 2013. In January 2009, a $2.0 \times 3.0 \mathrm{~cm}$ painless mass was surgically removed from the left parotid gland of the patient. Postoperative pathology characterized the mass as a lymphocytic hyperplasia; however, parotid duct cells were also detected within the lymphocytes. Morphological results and immunohistochemical staining supported the diagnosis of a BLEL.

$B L E L$ recurrence and long term development. After six months, the left parotid gland became locally swollen again and intermittent management with traditional Chinese medicine was unable to achieve remission. In October 2010, the size of the mass reached $5.0 \times 6.0 \mathrm{~cm}$. A computerized tomography (CT) scan of the parotid gland indicated a soft tissue mass in the left neck area without uniform density or a clear border with the left parotid gland, and evident infiltration in the surrounding tissue. The mass was considered to be a recurrence of the BLEL in the left parotid gland; however, the patient did not agree to further surgical therapy. The mass in the left parotid gland area became gradually enlarged, and was more evident when the patient became ill with a cold; 
Table I. Changes in the T cell subpopulations during DC-CIK adoptive cellular immunotherapy treatment.

\begin{tabular}{|c|c|c|c|c|c|}
\hline Analysis date & $\begin{array}{l}\text { Total T lymphocytes } \\
\qquad\left(\mathrm{CD}^{+}\right), \%\end{array}$ & $\begin{array}{l}\text { Suppressor T cells }{ }^{\mathrm{b}} \\
\left(\mathrm{CD}^{+} \mathrm{CD}^{+}\right), \%\end{array}$ & $\begin{array}{l}\text { Inhibitory } \mathrm{T} \text { cells }{ }^{\mathrm{c}} \\
\left(\mathrm{CD} 3^{+} \mathrm{CD} 28^{-}\right), \%\end{array}$ & $\begin{array}{l}\text { Regulatory T cells } \\
\left(\mathrm{CD} 4^{+} \mathrm{CD} 25^{+}\right), \%\end{array}$ & $\begin{array}{l}\text { Immune status } \\
\left(\mathrm{CD} 4^{+} / \mathrm{CD}^{+}{ }^{+} \text {ratio }\right)\end{array}$ \\
\hline Jun 82012 & 79.2 & 59.3 & 32.2 & 0.7 & 0.42 \\
\hline Jun 282012 & 55.7 & 38.6 & 28.8 & 1.2 & 0.47 \\
\hline Jul 172012 & 35.7 & 18.1 & 14.8 & 1.0 & 0.87 \\
\hline Sep 52012 & 54.3 & 30.1 & 20.8 & 1.7 & 0.68 \\
\hline
\end{tabular}

Normal ranges: ${ }^{\mathrm{a}} 62.5-79.5 \%$; ${ }^{\mathrm{b}} 19.9-34.5 \%$; ${ }^{\mathrm{c}} 9.8-21.8 \%$; ${ }^{\mathrm{d}} 1.36-5.48 \%$; ${ }^{\mathrm{e}} 1.20-2.76 \%$. DC-CIK, dendritic cell-cytokine-induced killer cell.

however, the mass was slightly reduced following anti-infective treatment (mainly with cephalosporins). The patient also received traditional Chinese medicine for six months and local acupuncture therapy for 40 days. However, the mass in the left parotid gland area was continually increasing in size and reached a diameter of $15 \mathrm{~cm}$. On April 27 2013, the patient developed a fever of up to $40^{\circ} \mathrm{C}$, accompanied with shivering, weakness and a loss of appetite, which was more severe in the afternoon and at night. Remission was observed following self-medication with oral ibuprofen; however, the temperature of the patient increased after several hours and a light yellow, clear liquid was discharged from the ulceration on the skin surface of the left parotid gland mass. On May 4 2013, the patient visited the Jilin Cancer Hospital, where the local ulceration and infection of the left parotid gland mass was managed with anti-infective treatment for three days; however, the high temperature of the patient did not decrease and the mass did not reduce in size. The patient was admitted to the hospital for further therapy on May 8 2013, and physical examination identified a large mass of $17 \times 14 \mathrm{~cm}$ on the left side of the patient's face, a red and swollen skin surface and multiple ruptures in the middle of the mass with a light yellow liquid discharge. The peripheral skin of the mass was tenacious and swollen, and the skin temperature was high. A blood routine examination and liver and renal functions were found to be normal. Color ultrasound revealed a visible $18.2 \times 8.5-\mathrm{cm}$ low-echo area with strong-echo stripes under the skin on the left side of the patient's face, without the appearance of a clear margin. Color Doppler flow imaging revealed an abundant blood flow in the mass and the absence of normal parotid gland tissue, indicating a solid space-occupying lesion on the left parotid gland. A CT scan of the left parotid gland revealed an abnormal mass in a low-density image and an undefined margin of the lesion, with the superior border extending towards the orbit, the inferior border at the submaxillary level, the inner border at the left parapharyngeal space and the outside border at the skin of the left-side of the face. The mass reached $13.4 \times 9.7 \mathrm{~cm}$ in an axial view, showing uniform density and a CT value of $47 \mathrm{HU}$ (Fig. 1A), with the left parotid gland unable to be distinguished. The lesion was wrapped around the left sphenoid bone and mandible. In addition, morphological changes were observed, indicating recurrence of the BLEL in the left parotid gland with bone resorption of the sphenoid bone and mandible. A bacterial culture of the mass discharge indicated a Staphylococcus aureus infection, which was sensitive to levofloxacin. The body temperature of the patient returned to normal levels following the administration of levofloxacin for one week. A biopsy was not performed due to the weakness, high fever, local swelling and infection of the patient, as well as the abundant blood flow detected in the mass with color ultrasound. No consensus was reached with regard to further surgical intervention. Drugs inhibiting vascular endothelial growth (Endostar and Shenyi capsules) were administered for five days, but were discontinued by the patient due to local pain and swelling. Chemoradiotherapy was subsequently considered; however, due to the young age of the patient and the examination results not indicating malignance, the patient did not agree to the therapy.

Dendritic cell-cytokine-induced killer cell (DC-CIK) biotherapy. Following failed conservative treatment, DC-CIK therapy was initiated based on the immune index result of the patient, which found the percentage of inhibitory $\mathrm{T}$ cells to be $32.2 \%\left(\mathrm{CD}^{+} \mathrm{CD} 28\right.$; normal range, $\left.9.8-21.8 \%\right)$ and the $\mathrm{CD}^{+} / \mathrm{CD}^{+}$ratio to be 0.42 (Table I), indicating low immunity. The patient signed informed consent for the treatment protocol, which was approved for tumor patients by the Health Department of Jilin Province (Changchun, China) and the Ethics Committee of the Jilin Cancer Hospital.

Source of cells. Antigen-presenting DCs and CIK cells were produced using heat shock protein-70, extracted from a head and neck squamous carcinoma cell strain supplied by the Jilin Cancer Hospital, and cocultured with cord blood DCs.

DC-CIK protocol. The duration of the first treatment session was 10 days, after which the treatment was discontinued for 10 days. Following the initial treatment course, the lesion in the left parotid gland area was reduced, while the local infection and skin ulceration were soothed. Biotherapy was continued for three months (four sessions of treatment), after which the left parotid gland lesion disappeared and the skin ulceration was healed.

Treatment outcome. Parotid gland CT scans (Fig. 1B-D) indicated that the primary lesion of the left parotid gland area disappeared gradually and the bone destruction was completely recovered. The patient was discharged from hospital and the condition remained stable without signs of recurrence during a follow-up period of 10 months; however, the patient remains under close observation. 
A

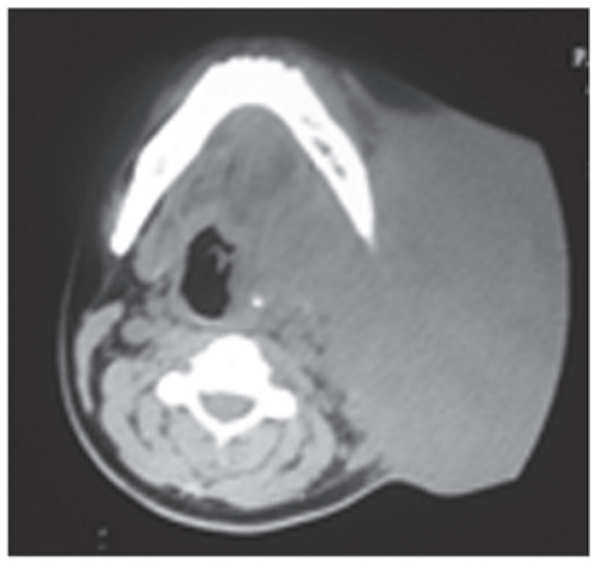

C

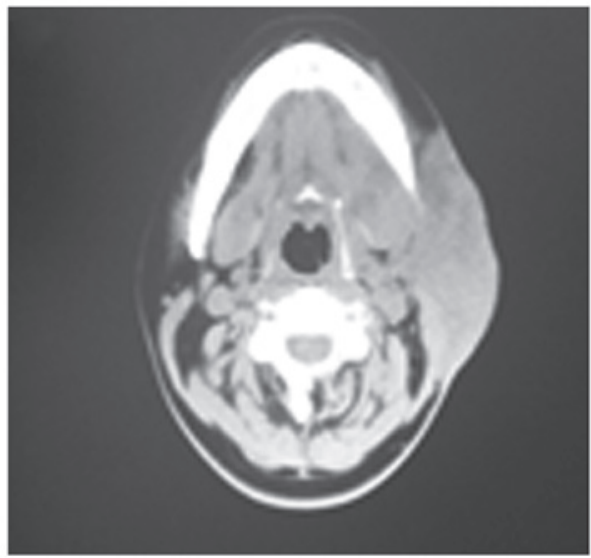

B

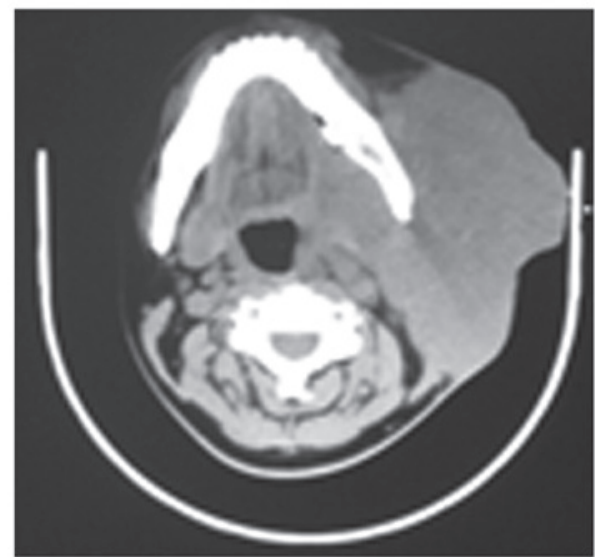

D

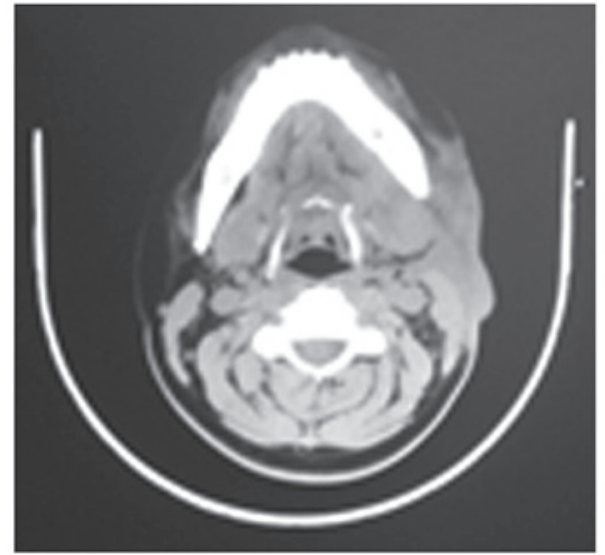

Figure 1. Computerized tomography images of the benign lymphoepithelial lesion in the left parotid gland (A) prior to treatment on May 8 2012, during the treatment on (B) Jun 122012 and (C) July 16 2012, and (D) after treatment on Sep 52012.

\section{Discussion}

BLELs, also known as Mikulicz disease (MD), were first reported by Mikulicz in 1888 and are characterized by chronic, painless and symmetric swelling of tear and salivary glands (10-12). When a BLEL is combined with certain systematic diseases, including sarcoidosis leukemia, viral infections and macroglobulinemia, the condition is also known as Mikulicz syndrome. In 1933, the Swedish optician, Sjögren, identified a chronic systematic disease, known as Sjögren's syndrome (SS), which primarily involved the salivary and tear glands (13). SS manifests as a dry mouth and xerotic keratitis, and occasionally occurs alongside other chronic connective tissue diseases. Due to the similar histological and pathological findings in MD and SS patients, Morgan and Raven proposed that MD is a subdisease of SS (14). Mihas et al hypothesized that MD, BLEL and SS may be conditions of the same disease process, with malignant lymphoma being a common complication (15). Due to the symptom similarities, the diagnosis of a BLEL is mainly based on clinical observations. An homogeneous, painless mass with a clear boundary at the tear gland, the absence of bone destruction and accompanied by a swollen salivary gland and dry mouth, can be diagnosed as a BLEL. However, prior to BLEL diagnosis, dry eyes, systematic connective tissue diseases and lymphoma and inflammatory pseudotumors should be excluded. In addition, diffuse hyperplasia and lymphocyte infiltration of the gland, atrophy of the gland parenchyma and the formation of epimyoepithelial islands in the gland duct should be pathologically confirmed (4).

The etiology of BLELs remains unclear. Tsubota et al revealed that although the pathologies of MD and SS are similar, the lacrimal gland acinar cells in SS patients were apoptotic with high protein expression levels of Fas and FasL, in contrast to the acinar cells of MD patients (16). In addition, Yamamoto et al observed that the serum concentration of the IgG4 antibody in BLEL patients was much higher when compared with the SS patients, and hypothesized that BLELs are a systematic disease correlating with IgG4 (17). Gupta et al revealed that the BLEL epimyoepithelial islands were predominantly surrounded by B lymphocytes and partially by $\mathrm{T}$ lymphocytes, indicating that humoral immunity was involved in BLELs (18). Initial T cell blood analysis of the patient in the present study revealed a high percentage of total $\mathrm{T}$ lymphocytes $\left(\mathrm{CD}^{+}\right)$, suppressor $\mathrm{T}$ cells $\left(\mathrm{CD}^{+} \mathrm{CD}^{+}\right)$ and inhibitory $\mathrm{T}$ cells $\left(\mathrm{CD}^{+} \mathrm{CD} 28^{-}\right)$, while a low $\mathrm{CD}^{+} / \mathrm{CD}^{+}$ ratio and number of regulatory $\mathrm{T}$ cells were observed. $\mathrm{CD}^{+} \mathrm{CD}^{+}$cells have been identified as malignant in $85 \%$ of granular lymphocyte leukemias (19), and previous studies have revealed that large granular lymphocyte leukemias are strongly associated with SS, in addition to other autoimmune diseases (20-22). A low $\mathrm{CD} 4^{+} / \mathrm{CD}^{+}$ratio, also observed in 
HIV-infected patients, is generally a sign of a weak immune system. Thus, in combination with the abnormally high percentages of inhibitory and suppressor T cells, the patient in the present study was considered to have a weakened immune system. In order to strengthen the patient's immunity, DC-CIK therapy was attempted, which had been previously used in the treatment of non-small cell lung cancer (23-25). As a result, the percentages of suppressor, inhibitory and regulatory $\mathrm{T}$ cells returned to within the normal ranges and the $\mathrm{CD}^{+} / \mathrm{CD}^{+}$ratio increased. However, the total $\mathrm{T}$ lymphocyte percentage decreased to below the normal range. Although the exact hematological mechanisms remain unclear and further analysis is required, considering the outcome of this first trial, DC-CIK therapy may be a promising approach for the treatment of BLELs.

\section{References}

1. Godwin JT: Benign lymphoepithelial lesion of the parotid gland adenolymphoma, chronic inflammation, lymphoepithelioma, lymphocytic tumor, Mikulicz disease. Cancer 5: 1089-1103, 1952.

2. Seifert G, Brocheriou C, Cardesa A and Eveson JW: WHO international histological classification of tumours. Tentative histological classification of salivary gland tumours. Pathol Res Pract 186: 555-581, 1990

3. Albrecht H, Stellbrink HJ and Greten H: Resolution of HIV-associated cystic benign lymphoepithelial lesion of the parotid gland in a patient undergoing chemotherapy for non-Hodgkin's lymphoma. Scand J Infect Dis 28: 621-623, 1996.

4. Tang DR, Shi XF, Sun FY, Zhao H and Jin YJ: Clinical features and therapy of benign lymphoepithelial lesion. Zhonghua Yan $\mathrm{Ke}$ Za Zhi 45: 441-445, 2009 (In Chinese).

5. Mourad WF, Hu KS, Shourbaji RA, Lin W and Harrison LB Radiation therapy for benign lymphoepithelial cysts of parotid glands in HIV patients. Laryngoscope 123: 1184-1189, 2013.

6. Hsiung CY, Huang CC, Wang CJ, Huang EY and Huang HY Lymphoepithelioma-like carcinoma of salivary glands: treatment results and failure patterns. Br J Radiol 79: 52-55, 2006

7. Sato K, Kawana M, Sato Y and Takahashi S: Malignant lymphoma in the head and neck associated with benign lymphoepithelial lesion of the parotid gland. Auris Nasus Larynx 29: 209-214, 2002

8. Schneider M and Rizzardi C: Lymphoepithelial carcinoma of the parotid glands and its relationship with benign lymphoepithelial lesions. Arch Pathol Lab Med 132: 278-282, 2008.

9. Ussmüller J, Reinecke T, Donath K and Jaehne M: Chronic myoepithelial sialadenitis - symptomatology, clinical signs, differential diagnostics. Laryngorhinootologie 81: 111-117, 2002 (In German).
10. Andrade RE, Hagen KA and Manivel JC: Distribution and immunophenotype of the inflammatory cell population in the benign lymphoepithelial lesion (Mikulicz's disease). Hum Pathol 19: 932-941, 1988

11. Sewkani A, Purohit D, Singh V, Jain A, Varshney R and Varshney S: Lymphoepithelial cyst of the pancreas: a rare case report and review of literature. Indian J Surg 72: 427-432, 2010.

12. Cho HJ, Kyung Myung J, Kim YH, Choi SH and Park SH: Benign lymphoepithelial tumor of the pituitary. Neuropathology 33: 413-417, 2013.

13. Sjögren H: Zur kenntis der keratoconjunctivitis sicca II Allgemeine Symptomatologie und Ätiologie. Acta Ophthalmol 13: 1-39, 1935.

14. Morgan AD and Raven RW: Sjögren's syndrome: a general disease. Br J Surg 40: 154-162, 1952.

15. Mihas AA, Lawson PB, Dreiling BJ, Gurram VS and Heuman DM: Mikulicz syndrome associated with a malignant large cell gastric lymphoma: a case report and review of the literature. Int J Gastrointest Cancer 33: 123-127, 2003.

16. Tsubota K, Fujita H, Tsuzaka K and Takeuchi T: Mikulicz's disease and Sjögren's syndrome. Invest Ophthalmol Vis Sci 41: 1666-1673, 2000

17. Yamamoto M, Naishiro Y, Suzuki C, et al: Proteomics analysis in 28 patients with systemic IgG4-related plasmacytic syndrome. Rheumatol Int 30: 565-568, 2010.

18. Gupta N, Gupta R, Rajwanshi A and Bakshi J: Multinucleated giant cells in HIV-associated benign lymphoepithelial cyst-like lesions of the parotid gland on FNAC. Diagn Cytopathol 37: 203-204, 2009

19. Rose MG and Berliner N: T-cell large granular lymphocyte leukemia and related disorders. Oncologist 9: 247-258, 2004.

20. Ergas D, Tsimanis A, Shtalrid M, Duskin C and Berrebi A: T-gamma large granular lymphocyte leukemia associated with amegakaryocytic thrombocytopenic purpura, Sjögren's syndrome, and polyglandular autoimmune syndrome type II, with subsequent development of pure red cell aplasia. Am J Hematol 69: 132-134, 2002.

21. Molad Y, Okon E, Stark P and Prokocimer M: Sjögren's syndrome associated T cell large granular lymphocyte leukemia: a possible common etiopathogenesis. J Rheumatol 28: 2551-2552, 2001.

22. Coakley G: Sjögren's syndrome associated T cell large granular lymphocyte leukemia: a possible common etiopathogenesis. J Rheumatol 29: 1803, 2002.

23. Shi SB, Ma TH, Li CH and Tang XY: Effect of maintenance therapy with dendritic cells: cytokine-induced killer cells in patients with advanced non-small cell lung cancer. Tumori 98: 314-319, 2012.

24. Li H, Wang C, Yu J, et al: Dendritic cell-activated cytokine-induced killer cells enhance the anti-tumor effect of chemotherapy on non-small cell lung cancer in patients after surgery. Cytotherapy 11: 1076-1083, 2009.

25. Yang L, Ren B, Li H, et al: Enhanced antitumor effects of DC-activated CIKs to chemotherapy treatment in a single cohort of advanced non-small-cell lung cancer patients. Cancer Immunol Immunother 62: 65-73, 2013. 